Pacific Journal of Mathematics

A RADON-NIKODYM THEOREM FOR FINITELY ADDITIVE 


\section{A RADON-NIKODYM THEOREM FOR FINITELY ADDITIVE SET FUNCTIONS}

\section{Charles FefFerman}

Suppose that $\Sigma$ is a field of subsets of the set $S$, and suppose that $\mu$ and $\gamma$ are complex-valued finitely additive set functions defined on $\Sigma$. Assume that $\mu$ is bounded and $\gamma$ is finite and absolutely continuous with respect to $\mu$. (A word of warning is in order here. The statement " $\gamma$ is absolutely continuous with respect to $\mu$ " is often interpreted as " $\mu(E)=0$ implies $\gamma(E)=0$ ". This is not the meaning used here. Our definition is "for every $\varepsilon>0$ there is a $\delta>0$ such that $|\mu(E)|<\delta$ implies $|\gamma(E)|<\varepsilon . "$ Unless $\mu$ is bounded and countably additive, the two definitions are not equivalent.)

Theorem 1. There exists a sequence $\left\{f_{n}\right\}$ of $\mu$-simple functions on $S$, such that

1.

$$
\lim _{n \rightarrow \infty} \int_{E} f_{n}(s) \mu(d s)=\gamma(E),
$$

uniformly for $E \in \Sigma$

2 .

$$
\lim _{n, m \rightarrow \infty} \int_{S}\left|f_{n}(s)-f_{m}(s)\right| v(\mu, d s)=0,
$$

where $v(\mu)$ is the total variation of $\mu$.

Theorem 1 is established by a pure existence proof, and gives no indication of how to find $f_{n}$. A more constructive result is given below.

A partition of $S$ is a finite collection of sets $E_{i}$ belonging to $\Sigma$, such that $S$ is the disjoint union of the $E_{i}$, and such that $\mu\left(E_{i}\right) \neq 0, i=1, \cdots, n$.

The set $\mathscr{P}$ of partitions may be directed by refinement, that is, by the following partial order: $P_{1}<P_{2}$ if for every $E \in P_{1}$ there exist $F_{1}, \cdots, F_{r} \in P_{2}(r$ may depend on $E)$ such that $E$ and $\bigcup_{i=1}^{r} F_{i}$ differ by a $\mu$-null set.

If $P$ is a partition of $S$, define the $\mu$-simple function $f_{P}^{\gamma}$ to be $\sum_{E \in_{P}}(\gamma(E) / \mu(E)) \chi_{E}$, where $\chi_{E}$ is the characteristic function of $E$.

Theorem 2. If $\mu$ is positive, then

$$
\lim _{P \in \mathscr{P}} \int_{E} f_{P}^{\gamma}(s) \mu(d s)=\gamma(E),
$$

uniformly for $E \in \Sigma$, where $\mathscr{P}$ is directed as explained above.

(The notation throughout is essentially that of [2].) For positive $\mu$, Theorem 1 reduces to Bochner's Radon-Nikodym Theorem. See [1]. 
Proofs of the theorems. Theorem 2 follows with little difficulty from Theorem 1. Therefore, for the sake of clarity, we shall first establish Theorem 2, assuming Theorem 1 to be valid.

Proof of Theorem 2. The result is proved by translating the situation into an abstract problem on the convergence of operators in a Banach space.

Throughout the proof, we assume $\gamma$ bounded. This assumption is justified by Lemma 1 in the proof of Theorem 1, which shows that boundedness of $\gamma$ follows from the hypothesis of Theorem 2 .

Let $X$ be the vector space of bounded $\mu$-absolutely continuous set functions on $\Sigma$ with the norm $|\gamma|=v(\gamma, S)$. Define continuous linear operators $T_{P}$ on $X, P \in \mathscr{P}$, by the relation

$$
\left(T_{P}(\gamma)\right)(E)=\int_{E} f_{P}^{\gamma}(s) \mu(d s) .
$$

Theorem 2 asserts that for each $\gamma \in X, \lim _{P \in \mathscr{P}} T_{P}(\gamma)=\gamma$. In order to show this, it suffices to prove

(1) that $\lim _{P \in \mathscr{P}} T_{P}(\gamma)=\gamma$ for all $\gamma$ in a dense subset of $\gamma$;

(2) that the operators $T_{P}, P \in \mathscr{P}$ are equicontinuous on the unit ball of $X$; that is, $\left|T_{P}\right|, P \in \mathscr{P}$ are uniformly bounded.

To establish (1), we select as our dense subset the set of all $\gamma \in X$ of the form

$$
\gamma(E)=\int_{E} f(s) \mu(d s),
$$

where $f$ is a $\mu$-simple function on $S$. That this subset is dense follows from Theorem 1. We must show that if $\gamma$ is of the above form, then $\lim _{P \in \mathscr{P}} T_{P}(\gamma)=\gamma$. But this is immediate from the following argument: Let

$$
\gamma(E)=\int_{E} f(s) \mu(d s),
$$

$E \in \Sigma$, where (since $f$ is a $\mu$-simple function), we can write

$$
f=\sum_{i=1}^{N} \alpha_{i} \chi_{E_{i}}
$$

with the $E_{i}$ pairwise disjoint, and $S=\bigcup_{i=1}^{N} E_{i}$. Then $\left\{E_{i}\right\}$ is a partition of $S$. By trivial algebra,

$$
T_{P}(\gamma)=\int_{(\cdot)} f_{P}^{\gamma}(s) \mu(d s)=\gamma
$$

whenever $P$ is a refinement of $\left\{E_{i}\right\}$, that is, $\left\{E_{i}\right\}>P$. This proves (1). 
To establish (2), we shall prove that $\left|T_{P}\right| \leqq 1, P \in \mathscr{P}$, i.e., $\left|T_{P} \gamma\right| \leqq|\gamma|, \gamma \in X, P \in \mathscr{P}$. Any $\gamma \in X$ may be separated into its real and complex parts, and so it suffices to show that $\left|T_{P} \gamma\right| \leqq|\gamma|$ when $\gamma$ is real. Since any real $\gamma$ is the difference of its positive and negative parts, $\gamma=\left(\gamma_{+}\right)-\left(\gamma_{-}\right)$, and since $\left|\gamma_{+}\right|+\left|\gamma_{-}\right|=|\gamma|$ (by definition of the norm in $X)$, it suffices to show that $\left|T_{P} \gamma\right| \leqq \gamma$ when $\gamma$ is positive, and $P \in \mathscr{P}$. Now we must show that if $\gamma$ is positive, and $P \in \mathscr{P}$, then $\left|T_{P} \gamma\right| \leqq|\gamma|=v(\gamma, S)=\gamma(S)$. By inspection of the definition of $T_{P}$, we see that since $\mu$ and $\gamma$ are positive, $T_{P} \gamma$ is positive. So we must show that $\left(T_{P} \gamma\right)(S) \leqq \gamma(S)$, when $\gamma$ is positive. By trivial algebra, it follows from the definition of $T_{P}$ that $\left(T_{P} \gamma\right)(S)=\gamma(S)$.

This proves Theorem 2 .

We can now proceed to the most important part of the paper.

Proof of Theorem 1. The plan of attack is as follows: First we prove the theorem for the case in which $\mu$ is positive, and there is an $N>0$ such that $|\gamma(E)| \leqq N \mu(E), E \in \Sigma$. Next, we remove the restriction on $\gamma$, and show that the theorem holds for all positive $\mu$. Finally, we extend to the general case of a complex $\mu$, using the approach of [2].

Let us establish a simplified notation. If $\gamma$ is any bounded $\mu$-absolutely continuous set function on $\Sigma$, and $f$ is a $\mu$-simple function on $S$, define the $\mu$-absolutely continuous finitely additive set function $f \gamma$ by the relation

$$
(f \gamma)(E)=\int_{E} f(s) \gamma(d s)
$$

This operation makes $X$, the space defined earlier, a module over the set of $\mu$-simple functions on $S$.

This notation allows us to reformulate Theorem 1 in terms of the space $X$. Conclusion (1) states that $\lim _{n \rightarrow \infty} f_{n} \mu=\gamma$ in $X$, that is, $\lim _{n \rightarrow \infty} v\left(\gamma-f_{n} \mu, S\right)=0$. Conclusion (2) asserts that the sequence $\left\{f_{n} \mu\right\}$ is Cauchy in the $X$-norm. Since convergent sequence in $X$ are Cauchy, conclusion (2) follows from conclusion (1).

The fact that $\lim _{n \rightarrow \infty} v\left(\gamma-f_{n} \mu, S\right)=0$ if and only if conclusion (1) of the theorem holds, should be noted carefully, since this is the form in which the theorem will be proved.

We now present the series of lemmas which establishes Theorem 1.

LEMMA 1. Let $\mu$ and $\gamma$ be finite real-valued finitely additive set functions on $\Sigma$. Suppose that $\mu$ is bounded and positive, and $\gamma$ is r-absolutely continuous. Then $\gamma$ is bounded. 
Proof. Suppose not. Then $\mathscr{C}=\{E \in \Sigma \mid v(\gamma, E)=+\infty\}$ is nonempty. Let $M=\inf \{\mu(E) \mid E \in \mathscr{C}\}$. Pick a $\delta>0$ so small that $\mu(E)<\delta$ implies $|\gamma(E)| \leqq 1$. Pick an $E_{0} \in \mathscr{C l}$ such that $\mu\left(E_{0}\right)<M+(\delta / 2)$. Since $E_{0} \in \mathscr{L}, v(\gamma, E)=+\infty$. On the other hand, we shall show that for every $F \in \Sigma, F \subseteq E_{0},|\gamma(F)|+\left|\gamma\left(E_{0}-F\right)\right| \leqq\left|\gamma\left(E_{0}\right)\right|+2$. This will be a contradiction, since, $\gamma$ being real,

$$
v\left(\gamma, E_{0}\right)=\operatorname{Sup}_{\substack{F \in \Sigma \\ F \cong E_{0}}}\left(|\gamma(F)|+\left|\gamma\left(E_{0}-F\right)\right|\right) \leqq\left|\gamma\left(E_{0}\right)\right|+2<+\infty .
$$

So to establish Lemma 1, we need only show that if $F \in \Sigma$, $F \cong E_{0}$, then $|\gamma(F)|+\left|\gamma\left(E_{0}-F\right)\right| \leqq\left|\gamma\left(E_{0}\right)\right|+2$. Pick an $F \in \Sigma$, $F \cong E_{0}$. If we had $\mu(F) \geqq \delta, \mu\left(E_{0}-F\right) \geqq \delta$, then by finite additivity,

$$
\begin{aligned}
\mu(F) & =\mu\left(E_{0}\right)-\mu\left(E_{0}-F\right) \\
& <\left(M+\frac{\delta}{2}\right)-\mu\left(E_{0}-F\right) \leqq\left(M+\frac{\delta}{2}\right)-\delta<M \\
\mu\left(E_{0}-F\right) & =\mu\left(E_{0}\right)-\mu(F) \\
& <\left(M+\frac{\delta}{2}\right)-\mu(F) \leqq\left(M+\frac{\delta}{2}\right)-\delta<M
\end{aligned}
$$

so that, by definition of $M, F \notin \mathscr{C l}$ and $E-F \notin \mathscr{C}$. But then, $v(\gamma, F)<+\infty$ and $v\left(\gamma, E_{0}-F\right)<+\infty$, so that $v\left(\gamma, E_{0}\right)<+\infty$, contradicting $E_{0} \in \mathscr{L}$.

Therefore, if $F \in \Sigma, F \subseteq E_{0}$, then either $\mu(F)<\delta$ or $\mu\left(E_{0}-F\right)<\delta$. Suppose that $\mu(F)<\delta$. Then by definition of $\delta,|\gamma(F)| \leqq 1$. On the other hand,

$$
\begin{aligned}
\left|\gamma\left(E_{0}-F\right)\right| & =\left|\gamma\left(E_{0}\right)-\gamma(F)\right| \\
& \leqq\left|\gamma\left(E_{0}\right)\right|+|\gamma(F)| \leqq\left|\gamma\left(E_{0}\right)\right|+1 .
\end{aligned}
$$

Therefore,

$$
|\gamma(F)|+\left|\gamma\left(E_{0}-F\right)\right| \leqq\left|\gamma\left(E_{0}\right)\right|+2 \text {. }
$$

Similar arguments show that if $\mu\left(E_{0}-F\right)<\delta$, then

$$
|\gamma(F)|+\left|\gamma\left(E_{0}-F\right)\right| \leqq\left|\gamma\left(E_{0}\right)\right|+2 \text {. }
$$

So whenever $F \in \Sigma, F \subseteq E_{0},|\gamma(F)|+\left|\gamma\left(E_{0}-F\right)\right| \leqq\left|\gamma\left(E_{0}\right)\right|+2$.

LEMMA 2. Let $\mu$ be a positive bounded finitely additive set function on $\Sigma$, and let $\gamma$ be a real-valued finite, finitely additive set function on $\Sigma$. If there is an $N>0$ such that $|\gamma(E)| \leqq N \mu(E)$ for all $E \in \Sigma$, then there exists a sequence $\left\{f_{n}\right\}$ of $\mu$-simple functions on $S$, such that 


$$
\gamma(E)=\lim _{n \rightarrow \infty} \int_{E} f_{n}(s) \mu(d s)
$$

uniformly for $E \in \Sigma$.

Proof. Let $\mathscr{L}$ be the space of $\mu$-simple functions on $S$, with the norm

$$
|f|=\sqrt{\int_{s}(f(s))^{2} \mu(d s)}
$$

and let $\overline{\mathscr{L}}$ be the completion of $\mathscr{L}$. $\overline{\mathscr{L}}$ is obviously a Hilbert space. For example, if $\mu$ is countably additive, then $\overline{\mathscr{L}}$ is just the space $L_{2}$. Define a linear functional $\mathscr{O}$ on $\mathscr{L}$ as follows:

$$
\mathscr{O} f=\int_{s} f(s) \gamma(d s)
$$

To check that $\mathcal{O}$ is continuous, observe that

$$
|\mathscr{O} f| \leqq \int_{S}|f(s)| v(\gamma, d s) \leqq N \int_{S}|f(s)| \mu(d s) \leqq N|f| \cdot|1|
$$

where 1 is the $\mu$-simple function which maps all of $S$ to 1 . (The last inequality is just Hölder's inequality with $g=1$.)

Since $\mathscr{O}$ is continuous on $\mathscr{L}$, it has a continuous extension $\bar{O}$ to all of $\overline{\mathscr{S}}$. Since $\overline{\mathscr{O}}$ is a continuous linear functional on a Hilbert space, there is some $x \in \overline{\mathscr{L}}$ such that for all $y \in \overline{\mathscr{L}}, \bar{O} y=x \cdot y$. Since $\mathscr{L}$ is dense in $\overline{\mathscr{L}}$, there exists a sequence $\left\{f_{n}\right\}$ of elements of $\mathscr{L}$ (i.e., a sequence of $\mu$-simple functions on $S$ ) such that $\lim _{n \rightarrow \infty} f_{n}=x$ in $\overline{\mathscr{L}}$. Therefore, for each positive $M, \lim _{n \rightarrow \infty} f_{n} \cdot y=$ of uniformly for $y$ in the $M$-ball of $\overline{\mathscr{L}}$.

For $E \in \Sigma,\left|\chi_{E}\right|$ in $\mathscr{L}$ is just

$$
\begin{aligned}
\left|\chi_{E}\right| & =\sqrt{\int_{S}\left(\chi_{E}(s)\right)^{2} \mu(d s)} \\
& =\sqrt{\int_{E} 1 \mu(d s)}=\sqrt{\mu(E)} \leqq \sqrt{\mu(S)} .
\end{aligned}
$$

Therefore, $\left\{\chi_{E} \mid E \in \Sigma\right\}$ consists entirely of elements of the $M$-ball of $\overline{\mathscr{L}}$ where $M=\sqrt{\mu(S)}$. By the result of the last paragraph,

$$
\lim _{n \rightarrow \infty} f_{n} \cdot \chi_{E}=\mathscr{O} \chi_{E},
$$

uniformly for $E \in \Sigma$. Recalling the definitions of $\mathcal{O}$ and of the dot product in $\mathscr{L}$, this equation becomes 


$$
\lim _{n \rightarrow \infty} \int_{S} f_{n}(s) \chi_{E}(s) \mu(d s)=\int_{S} \chi_{E}(s) \gamma(d s)
$$

uniformly for $E \in \Sigma$, i.e.

$$
\lim _{n \rightarrow \infty} \int_{E} f_{n}(s) \mu(d s)=\gamma(E)
$$

uniformly for $E \in \Sigma$.

The next step in the proof of Theorem 1 is to extend Lemma 2 to the case in which we no longer have $|\gamma(E)| \leqq N \mu(E)$. It becomes easier to see how this extension may be accomplished, if we rephrase the conclusion that we are trying to prove. We are trying to show that if $\mu$ is as in Lemma 2, then $\{f \mu \mid f$ a $\mu$-simple function on $S\}$ is dense in $X$. By Lemma 2, $\{f \mu \mid f$ a $\mu$-simple function on $S\}$ is dense in $X_{0}=\{\gamma \in X|\exists N>0 \ni \forall E \in \Sigma,| \gamma(E) \mid \leqq N \mu(E)\}$. So we want to show that $X_{0}$ is dense in $X$, i.e., for every $\mu$-absolutely continuous finite $\gamma$, there is a sequence $\gamma_{n}$ of set functions of $X$, such that $\left\{\gamma_{n}(E) \mid \leqq n \mu(E), E \in \Sigma\right.$, and $\lim _{n \rightarrow \infty} v\left(\gamma-\gamma_{n}, S\right)=0$. We find $\left\{\gamma_{n}\right\}$ as follows:

DEFINITION. Let $\gamma_{1}$ and $\gamma_{2}$ be finite positive finitely additive set functions on $\Sigma$. Define the set function $\gamma_{1} \wedge \gamma_{2}$ on $\Sigma$ by the relation

$$
\left(\gamma_{1} \wedge \gamma_{2}\right)(E)=\inf _{\substack{F \in \Sigma \\ F=E}}\left[\gamma_{1}(F)+\gamma_{2}(E-F)\right]
$$

Observations.

1. $\gamma_{1} \wedge \gamma_{2}$ is finitely additive. This is easy, and the proof is contained in the proof of Theorem III 7.3 in [2]. Therefore the proof will not be included here.

2. $\gamma_{1} \wedge \gamma_{2} \leqq \gamma_{1}$ and $\gamma_{1} \wedge \gamma_{2} \leqq \gamma_{2}$.

3 . If $\gamma_{2} \leqq \gamma_{3}$ then $\gamma_{1} \wedge \gamma_{2} \leqq \gamma_{1} \wedge \gamma_{3}$.

LEMma 3. Let $\mu$ be a positive bounded finitely additive set function on $\Sigma$, and let $\gamma$ be a positive bounded finitely additive $\mu$-absolutely continuous set function on $\Sigma$. Let $\gamma_{n}=(n \mu) \wedge \gamma$. Then

$$
\lim _{n \rightarrow \infty} v\left(\gamma-\gamma_{n}, S\right)=0 \text {. }
$$

Proof. $0 \leqq \gamma_{n} \leqq \gamma_{n+1} \leqq \gamma$, so that if $\lim _{n \rightarrow \infty} \gamma_{n}(S)=\gamma(S)$, the lemma is proved. Let $\varepsilon>0$ be given. By hypothesis, there is a $\delta>0$ such that if $\mu(E)<\delta, E \in \Sigma$, then $\gamma(E) \leqq \varepsilon$. Let $N \geqq \gamma(S) / \delta$. Consider any $F \in \Sigma$.

Case 1. $\mu(F)>\delta$. Then $N \mu(F) \geqq \gamma(S)$, so that 


$$
N \mu(F)+\gamma(S-F) \geqq \gamma(S) .
$$

Case 2. $\mu(F)<\delta$. Then $\gamma(F) \leqq \varepsilon$, so that $\gamma(S-F) \geqq \gamma(S)-\varepsilon$, and so

$$
N \mu(F)+\gamma(S-F) \geqq \gamma(S)-\varepsilon .
$$

In either case, $N \mu(F)+\gamma(S-F) \geqq \gamma(S)-\varepsilon$. So

$$
\gamma_{N}(S)=\inf _{F \in \Sigma}(N \mu(F)+\gamma(S-F)) \geqq \gamma(S)-\varepsilon .
$$

Therefore, $\lim _{n \rightarrow \infty} \gamma_{n}(S)=\gamma(S)$.

LEMMA 4. Let $\mu$ be a positive bounded finitely additive set function on $\Sigma$, and let $\gamma$ be a complex-valued finite $\mu$-absolutely continuous finitely additive set function on $\Sigma$. Then there exists a sequence $\left\{f_{n}\right\}$ of $\mu$-simple functions on $S$, such that

$$
\lim _{n \rightarrow \infty} v\left(\gamma-f_{n} \mu, S\right)=0 \text {. }
$$

Proof. $\gamma$ can be separated into its real and imaginary parts, so that it suffices to consider $\gamma$ real. Any real $\gamma$ may be separated into its positive and negative parts, $\gamma=\gamma_{+}-\gamma_{-}$. Lemma 1 assures us that $\gamma_{+}$and $\gamma_{-}$are bounded. So it suffices to consider $\gamma$ positive. For $n>0$, pick $N_{n}$ so large that

$$
v\left(\gamma-\left(N_{n} \mu \wedge \gamma\right), S\right)<\frac{1}{2 n} .
$$

This is possible by Lemma 3. By Lemma 2, and by the fact that $\left|\left(N_{n} \mu \wedge \gamma\right)(E)\right| \leqq N_{n} \mu(E), E \in \Sigma$, there is an $f_{n}$, a $\mu$-simple function on $S$, such that

$$
v\left(\left(N_{n} \mu \wedge \gamma\right)-f_{n} \mu, S\right)<\frac{1}{2 n} .
$$

Then

$$
\begin{aligned}
v\left(\gamma-f_{n} \mu, S\right) & \leqq v\left(\left(\gamma-N_{n} \mu \wedge \gamma\right), S\right)+v\left(\left(N_{n} \mu \wedge \gamma\right)-f_{n} \ell, S\right) \\
& <\frac{1}{2 n}+\frac{1}{2 n}=\frac{1}{n},
\end{aligned}
$$

so that $\lim _{n \rightarrow \infty} v\left(\gamma-f_{n} \mu, S\right)=0$.

We have now completed the second step in the proof of Theorem 1 , namely, we have proved the theorem for the case $\mu \geqq 0$. The final step is to establish the theorem in the complex case. To accomplish 
this, we carry over the procedure of [2], which is valid for the countably additive case. In [2], the argument (to prove the usual Radon-Nikodym Theorem in the complex case, given its validity in the case $\mu \geqq 0$ ) is roughly the following: Let $\mu$ and $\gamma$ be bounded measures on the sigma-field $\Sigma$, and suppose that $\gamma$ is $\mu$-absolutely continuous. Then $\gamma$ and $\mu$ are both $v(\mu, \cdot)$-absolutely continuous. So there are integrable functions $g$ and $h$ on $S$ such that

$$
\mu(E)=\int_{E} h(s) v(\mu, d s)
$$

and

$$
\gamma(E)=\int_{E} g(s) v(\mu, d s)
$$

$E \in \Sigma$. It is then proved that

$$
v(\mu, E)=\int_{E} \frac{1}{h(s)} \mu(d s),
$$

$E \in \Sigma$, and from this it is shown that

$$
\gamma(E)=\int_{E} \frac{g(s)}{h(s)} \mu(d s) .
$$

In order to apply this type of argument to the present situation, we need two estimates:

LEMma 5. Let $\mu$ be any bounded complex-valued finitely additive set function on $\Sigma$, and let $u$ denote the set function $v(\mu, \cdot)$. Then there exists a sequence $\left\{f_{n}\right\}$ of $\mu$-simple functions on $S$, such that $\left|f_{n}(s)\right| \geqq 1 / 2$ for $n>0$ and $s \in S$, and such that

$$
\lim _{n \rightarrow \infty} v\left(\mu-f_{n} v, S\right)=0 .
$$

Proof. By Lemma 2, there is a sequence $\left\{g_{n}\right\}$ of $\mu$-simple functions on $S$, such that $\lim _{n \rightarrow \infty} v\left(\mu-g_{n} u, S\right)=0$. Then

$$
\lim _{n \rightarrow \infty}\left|v(\mu, E)-v\left(g_{n} u, E\right)\right|=0
$$

uniformly in $E \in \Sigma$. Now

$$
v\left(g_{n} u, E\right)=\int_{E}\left|g_{n}(s)\right| v(\mu, d s),
$$

so that 


$$
\begin{aligned}
\left|v(\mu, E)-v\left(g_{n} u, E\right)\right| & =\left|v(\mu, E)-\int_{E}\right| g_{n}(s)|v(\mu, d s)| \\
& =\left|\int_{E}\left(1-\left|g_{n}(s)\right|\right) v(\mu, d s)\right| .
\end{aligned}
$$

Therefore,

$$
\lim _{n \rightarrow \infty} \int_{E}\left(1-\left|g_{n}(s)\right|\right) v(\mu, d s)=0
$$

uniformly for $E \in \Sigma$. Hence,

$$
\lim _{n \rightarrow \infty} \int_{s}|1-| g_{n}(s)|| v(\mu, d s)=0 .
$$

If $\left|g_{n}(s)\right|<1 / 2$, then $|1-| g_{n}(s)||>1 / 2$. Therefore, setting

$$
\begin{gathered}
S_{n}=\left\{s \in S|| g_{n}(s) \mid<\frac{1}{2}\right\}, \\
\frac{1}{2} v\left(\mu,\left\{s \in S|| g_{n}(s) \mid<\frac{1}{2}\right\}\right) \leqq \int_{S_{n}}|1-| g_{n}(s)|| v(\mu, d s) \\
\leqq \int_{S}|1-| g_{n}(s)|| v(\mu, d s) .
\end{gathered}
$$

So $\lim _{n \rightarrow \infty} v\left(\mu, S_{n}\right)=0$. Let $\chi_{n}$ be the characteristic function of $S_{n}$. Then

$$
\lim _{n \rightarrow \infty} \int_{s}\left|\chi_{n}(s)\right| v(\mu, d s)=0
$$

Define $f_{n}=g_{n}+\chi_{n}$. By checking the two cases $\left|g_{n}(s)\right| \geqq 1 / 2$ and $\left|g_{n}(s)\right|<1 / 2$, we see that $\left|f_{n}(s)\right| \geqq 1 / 2, n>0, s \in S$. Now

$$
\begin{aligned}
v\left(f_{n} u-g_{n} u, S\right) & =v\left(\left(f_{n}-g_{n}\right) u, S\right) \\
& =v\left(\chi_{n} u, S\right)=\int_{S}\left|\chi_{n}(s)\right| v(\mu, d s) .
\end{aligned}
$$

Since $\lim _{n \rightarrow \infty} v\left(\mu-g_{n} u, S\right)=0$, we have $\lim _{n \rightarrow \infty} v\left(\mu-f_{n} u, S\right)=0$.

Lemma 6. Let $\mu$ and $u$ be as in the previous lemma. Then there exists a sequence $\left\{g_{n}\right\}$ of $\mu$-simple functions on $S$, such that

$$
\lim _{n \rightarrow \infty} v\left(u-g_{n} \mu, S\right)=0 \text {. }
$$

Proof. Let $\left\{f_{n}\right\}$ be a sequence of $\mu$-simple functions on $S$, as in the conclusion of the previous lemma, and define $g_{n}(s)=1 / f_{n}(s) \cdot g$ is well-defined, since $f_{n}(s) \neq 0$.

Now $g_{n} \mu=g_{n} f_{n} u+g_{n}\left[\mu-f_{n} u\right]=u+g_{n}\left[\mu-f_{n} u\right]$. Since 


$$
\left|f_{n}(s)\right| \geqq \frac{1}{2}, \quad\left|g_{n}(s)\right|=\frac{1}{\left|f_{n}(s)\right|} \leqq 2,
$$

so that $v\left(g_{n}\left[\mu-f_{n} u\right], S\right) \leqq 2 v\left(\mu-f_{n} u, S\right)$. But

$$
\lim _{n \rightarrow \infty} v\left(\mu-f_{n} u, S\right)=0 .
$$

So

$$
\lim _{n \rightarrow \infty} v\left(g_{n} \mu-u, S\right)=\lim _{n \rightarrow \infty} v\left(g_{n}\left[\mu-f_{n} u\right], S\right)=0 .
$$

Proof of Theorem 1. By Lemma 4 there is a sequence $\left\{h_{n}\right\}$ of $\mu$-simple functions on $S$, such that $\lim _{n \rightarrow \infty} v\left(\gamma-h_{n} u, S\right)=0$, where $u$, as usual, stands for $v(\mu, \cdot)$. By Lemma 6 , there is a sequence $\left\{g_{n}\right\}$ of $\mu$-simple functions on $S$ such that $\lim _{n \rightarrow \infty} v\left(u-g_{n} \mu, S\right)=0$. Now

$$
\gamma=h_{m} u+\left(\gamma-h_{m} u\right)=h_{m} g_{N} \mu+h_{m}\left(u-g_{N} \mu\right)+\left(\gamma-h_{m} u\right) .
$$

Given $n>0$, pick $m_{n}$ so large that $v\left(\gamma-h_{m_{n}} u, S\right)<1 / 2 n$. Since $h_{m_{n}}$ is $\mu$-simple, we can pick $M_{n}>0$ such that $\left|h_{m_{n}}(s)\right| \leqq M_{n}, s \in S$. Pick $N_{n}$ so large that $v\left(u-g_{N_{n}} \mu, S\right)<1 / 2 n M_{n}$. Then it follows that

$$
\begin{aligned}
v\left(\gamma-h_{m_{n}} g_{N_{n}} \mu, S\right) & \leqq v\left(h_{m_{n}}\left(u-g_{N_{n}} \mu\right), S\right)+v\left(\gamma-h_{m_{n}} u, S\right) \\
& \leqq M_{n} v\left(u-g_{N_{n}} \mu, S\right)+v\left(\gamma-h_{m_{n}} u, S\right) \\
& <M_{n} \cdot \frac{1}{2 n M_{n}}+\frac{1}{2 n}=\frac{1}{2 n}+\frac{1}{2 n}=\frac{1}{n} .
\end{aligned}
$$

So, if we define $f_{n}=h_{m_{n}} g_{N_{n}}$, then we have $v\left(\gamma-f_{n} \mu, S\right)<1 / n$. Therefore,

$$
\lim _{n \rightarrow \infty} v\left(\gamma-f_{n} \mu, S\right)=0
$$

Some examples. This section exhibits counterexamples to show that the principal hypothesis, boundedness of $\mu$, is needed to prove Theorem 1.

ExAmple 1. Let $S$ be the set of natural numbers, and let $\Sigma$ be the set of all subsets of $S$. Define a countably additive set function $\mu$ by $\mu(\{n\})=1$, and let $\gamma$ be any finitely additive set function on $\Sigma$ that is not countably additive. If $v(\mu, E)<1 / 2$, then $E$ must be empty, so that $\gamma(E)=0$. Therefore $\gamma$ is $\mu$-absolutely continuous. But if $\gamma$ were representable as in Theorem 1, then we could show that $\gamma$ is countably additive. 
ExAmple 2. Let $S$ and $\Sigma$ be as in Example 1. Define finite set functions $\mu$ and $\gamma$ finitely additive on $\Sigma$ by setting $\mu(\{n\})=1$, $\gamma(\{n\})=n$, and extending by a Zorn's lemma argument. $\mu$ and $\gamma$ are finite, but not bounded. By the reasoning of Example $1, \gamma$ is $\mu$ absolutely continuous. If $\gamma$ were representable in the form of Theorem 1, then we could write $\gamma=\gamma_{1}+\gamma_{2}$ where $v\left(\gamma_{1}, S\right)<1$ and $\left|\gamma_{2}(E)\right| \leqq M|\mu(E)|, E \in \Sigma$. In fact, the above decomposition of $\gamma$ is impossible.

\section{REFERENCES}

1. S. Bochner, Additive set functions on groups Ann. of Math. 40 (1939), 769-799.

2. N. Dunford and J. Schwartz, Linear Operators, Interscience, New York, 1958.

Received May 20, 1966. This work was performed under National Science Foundation Grant NSF GE-6214. 



\section{PACIFIC JOURNAL OF MATHEMATICS}

\section{EDITORS}

\section{H. ROYDEN}

Stanford University

Stanford, California

J. P. JANS

University of Washington

Seattle, Washington 98105

\section{J. DUGUNDJI}

Department of Mathematics

Rice University

Houston, Texas 77001

RICHARD ARENS

University of California

Los Angeles, California 90024

ASSOCIATE EDITORS

E. F. BECKENBACH

B. H. NeumanN

F. WoLF

K. YosidA

\section{SUPPORTING INSTITUTIONS}

\author{
UNIVERSITY OF BRITISH COLUMBIA \\ CALIFORNIA INSTITUTE OF TECHNOLOGY \\ UNIVERSITY OF CALIFORNIA \\ MONTANA STATE UNIVERSITY \\ UNIVERSITY OF NEVADA \\ NEW MEXICO STATE UNIVERSITY \\ OREGON STATE UNIVERSITY \\ UNIVERSITY OF OREGON \\ OSAKA UNIVERSITY \\ UNIVERSITY OF SOUTHERN CALIFORNIA
}

\author{
STANFORD UNIVERSITY \\ UNIVERSITY OF TOKYO \\ UNIVERSITY OF UTAH \\ WASHINGTON STATE UNIVERSITY \\ UNIVERSITY OF WASHINGTON \\ AMERICAN MATHEMATICAL SOCIETY \\ CHEVRON RESEARCH CORPORATION \\ TRW SYSTEMS \\ NAVAL ORDNANCE TEST STATION
}




\section{Pacific Journal of Mathematics}

\section{Vol. 23, No. 1 \\ March, 1967}

M. J. C. Baker, A spherical Helly-type theorem ................... 1

Robert Morgan Brooks, On locally m-convex*-algebras.............. 5

Lindsay Nathan Childs and Frank Rimi DeMeyer, On automorphisms of separable algebras ...................................

Charles L. Fefferman, A Radon-Nikodym theorem for finitely additive set

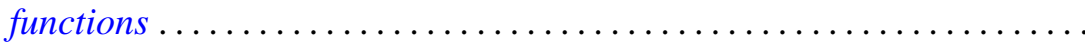

Magnus Giertz, On generalized elements with respect to linear

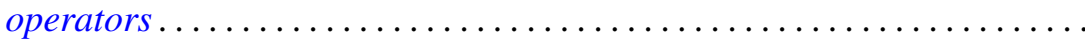

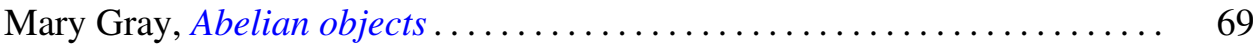

Mary Gray, Radical subcategories.............................. 79

John A. Hildebrant, On uniquely divisible semigroups on the two-cell . . . . . 91

Barry E. Johnson, AW*-algebras are $\mathrm{QW}^{*}$-algebras ............... 97

Carl W. Kohls, Decomposition spectra of rings of continuous functions . . . . 101

Calvin T. Long, Addition theorems for sets of integers .............. 107

Ralph David McWilliams, On $w^{*}$-sequential convergence and quasi-reflexivity ................................... 113

Alfred Richard Mitchell and Roger W. Mitchell, Disjoint basic

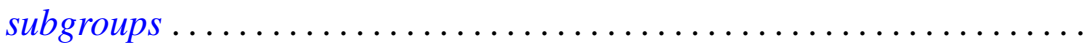

John Emanuel de Pillis, Linear transformations which preserve hermitian and positive semidefinite operators .

Qazi Ibadur Rahman and Q. G. Mohammad, Remarks on Schwarz's lemma

Neal Jules Rothman, An $L^{1}$ algebra for certain locally compact topological semigroups ...

F. Dennis Sentilles, Kernel representations of operators and their adjoints ...

D. R. Smart, Fixed points in a class of sets

K. Srinivasacharyulu, Topology of some Kähler manifolds

Francis C.Y. Tang, On uniqueness of generalized direct decompositions .

171 Albert Chapman Vosburg, On the relationship between Hausdorff dimension and metric dimension . 